\title{
Improving Adjuvant Endocrine Treatment Tailoring in Premenopausal Women With Hormone Receptor-Positive Breast Cancer
}

\author{
Matteo Lambertini, MD, $\mathrm{PhD}^{1,2}$; Eva Blondeaux, $\mathrm{MD}^{1}$; Francesco Perrone, $\mathrm{MD}^{3}$; and Lucia Del Mastro, MD ${ }^{1,2}$
}

The Oncology Grand Rounds series is designed to place original reports published in the Journal into clinical context. A case presentation is followed by a description of diagnostic and management challenges, a review of the relevant literature, and a summary of the authors' suggested management approaches. The goal of this series is to help readers better understand how to apply the results of key studies, including those published in Journal of Clinical Oncology, to patients seen in their own clinical practice.

\section{INTRODUCTION}

The Oncology Grand Rounds series is designed to place original reports published in the Journal of Clinical Oncology into clinical context. A case presentation is followed by a description of diagnostic and management challenges, a review of the relevant literature, and a summary of the authors' suggested management approaches. The goal of this series is to help readers better understand how to apply the results of key studies, including those published in $J C O$, to patients seen in their own clinical practice.

\section{CASE PRESENTATIONS}

A 41-year-old premenopausal woman was diagnosed with stage I breast cancer; she underwent lumpectomy and sentinel lymph node dissection for a left-sided breast cancer measuring $1.1 \mathrm{~cm}$. None of the two sentinel lymph nodes contained metastatic carcinoma. The tumor had ductal histology and was considered grade 1 of 3 . Immunohistochemical studies showed that the tumor was strongly positive for es-
ASSOCIATED CONTENT

See accompanying article doi 10.1200/ JC0.18.01967

Author affiliations and support applicable) appear at the end of this article.

Accepted on

September 13, 2019 and published at jco.org on October 16, 2019: DOI https://doi. org/10.1200/JC0.19. 02242

(C) 2019 by American Society of Clinical Oncology information (if trogen and progesterone receptor expression, was negative for human epidermal growth factor receptor 2 (HER2) overexpression, and had a Ki-67 score of 11\%.

A 39-year-old premenopausal woman was diagnosed with stage I breast cancer; she underwent mastectomy and sentinel lymph node dissection for a right-sided breast cancer measuring $1.1 \mathrm{~cm}$. The sentinel lymph node did not contain metastatic carcinoma. The tumor had ductal histology and was considered grade 2 of 3 . Immunohistochemical analysis showed HER2-negative disease with intermediate positivity for estrogen and progesterone receptor expression. A 21-gene assay was requested and returned a recurrence score (RS) of 21.

A 40-year-old premenopausal woman presented with stage II breast cancer; she underwent quadrantectomy and sentinel lymph node dissection for a left-sided breast cancer measuring $1.1 \mathrm{~cm}$. One of three sentinel lymph nodes contained metastatic carcinoma. The

tumor had ductal histology and was considered grade 2 of 3. Immunohistochemical studies showed HER2negative disease with intermediate positivity for estrogen and progesterone receptor expression. At another institution, adjuvant chemotherapy followed by radiation therapy (without axillary dissection) and endocrine therapy was recommended. After four cycles of adjuvant docetaxel and cyclophosphamide, she developed chemotherapy-induced amenorrhea; nevertheless, a blood test 1 month after chemotherapy completion showed estradiol and follicle-stimulating hormone (FSH) levels in the premenopausal range. She came to our attention seeking a second opinion to discuss the optimal adjuvant endocrine treatment.

A 39-year-old premenopausal woman was diagnosed with stage II breast cancer; she underwent mastectomy and axillary node dissection for a right-sided breast cancer measuring $2.3 \mathrm{~cm}$. Three of twelve axillary nodes contained metastatic carcinoma. The tumor had ductal histology and was considered grade 3 of 3. Immunohistochemical analysis showed HER2negative disease with high (95\%) and intermediate (40\%) positivity for estrogen and progesterone receptor expression, respectively.

All four clinical cases were discussed at our weekly multidisciplinary tumor board.

\section{CHALLENGES IN DIAGNOSIS AND MANAGEMENT}

Breast cancer is the most common malignancy in premenopausal women, ${ }^{1}$ accounting for more than $50 \%$ of all new occurrences in some countries. ${ }^{2}$ Despite higher chances of developing aggressive breast cancer subtypes, the majority of tumors in premenopausal patients have positive estrogen and progesterone receptor expression. ${ }^{3}$ Therefore, endocrine treatment is a mainstay adjuvant therapy for these patients. Optimal and refined estimation of prognosis and treatment benefit are key factors for choosing the best adjuvant treatment, which includes 
the possibility of adding chemotherapy in women with hormone receptor-positive tumors. ${ }^{4}$ This is of particular relevance for premenopausal patients, given the significant chemotherapy-related risk of long-term adverse effects with major impact on quality of life. ${ }^{5}$ These potential age-related negative consequences of chemotherapy include impairment of fertility and chances of future motherhood, which are highly relevant concerns that influence patient choices and adherence toward the recommended treatments and subsequently their disease-related outcomes. ${ }^{6-8}$

Age and menopausal status are crucial factors in the choice of the optimal adjuvant treatment. ${ }^{4}$ Recent data suggest that the known independent poor prognostic value of young age at diagnosis is restricted to hormone receptor-positive tumors. ${ }^{9-11}$ There are possible clinical and biologic explanations for these findings. First, these studies were conducted when tamoxifen alone was considered the standard of care as adjuvant endocrine treatment. ${ }^{12}$ Therefore, a significant proportion of premenopausal patients were undertreated according to current recommendations. ${ }^{13} \mathrm{Sec}-$ ond, young age is associated with suboptimal adherence to adjuvant endocrine treatment. ${ }^{14,15}$ Fertility and pregnancyrelated concerns represents key factors for noninitiation and early treatment discontinuation. ${ }^{16,17}$ Finally, there are potential biologic differences between breast cancers arising in young and older patients, ${ }^{18,19}$ particularly for hormone receptorpositive tumors. ${ }^{20}$ These data further highlight that specific attention should be paid to the management-including selection of the best adjuvant endocrine treatment—of hormone receptor-positive breast cancer in premenopausal women.

For many years, tamoxifen has been the standard adjuvant endocrine treatment option for premenopausal women with hormone receptor-positive tumors. ${ }^{12}$ In recent years, evidence on the role of ovarian function suppression (OFS) added to tamoxifen or to an aromatase inhibitor (AI) has radically changed the endocrine treatment landscape in this setting. ${ }^{13}$ Although a better understanding about how to integrate genomic information with classic clinicopathologic features has significantly improved our ability to estimate patient prognosis and chemotherapy benefit, ${ }^{21,22}$ adjuvant endocrine treatment tailoring in premenopausal women remains challenging. ${ }^{23,24}$ Therefore, having a new tool that could aid and engage patients and physicians during endocrine treatment decision making represents an important resource with a crucial impact on daily clinical practice.

\section{SUMMARY OF THE RELEVANT LITERATURE}

\section{Evolving Adjuvant Endocrine Treatment Landscape in Premenopausal Women}

The use of tamoxifen for 5 years significantly reduces the risk of breast cancer recurrence and death during the first 15 years after diagnosis in all patients with hormone receptor-positive tumors, irrespective of age at diagnosis. ${ }^{25}$
In premenopausal patients with hormone receptor-positive breast cancer, the development of chemotherapy-induced amenorrhea is associated with improved prognosis. ${ }^{26,27}$ Nevertheless, the benefit of temporary (ie, using a gonadotropin-releasing hormone agonist [GnRHa]) or permanent (ie bilateral oophorectomy or ovarian irradiation) OFS in addition to tamoxifen has remained unclear until recently. ${ }^{28}$ Three trials have now clarified the role of escalating adjuvant endocrine treatment by combining OFS with tamoxifen (Table 1)..$^{29-31}$ The E-3193 INT-0142 study did not show any difference in disease-free survival (DFS) or overall survival (OS) between patients treated with tamoxifen alone or combined with OFS for 5 years. ${ }^{29}$ Notably, this was a small trial that included low-risk patients (primary tumors $\leq 3 \mathrm{~cm}$, node-negative disease, no prior adjuvant chemotherapy allowed). Conversely, a significant DFS and OS improvement was observed by adding OFS to tamoxifen in the updated analysis of SOFT and in the ASTRRA study. ${ }^{30,31}$ SOFT was a three-arm trial in which premenopausal patients were randomly allocated to receive tamoxifen alone or combined with OFS or the combination of OFS plus exemestane. ${ }^{32}$ OFS was administered for 5 years; patients with or without exposure to prior chemotherapy were included, but those who received cytotoxic therapy (53\% of the study population) could be randomly assigned within 8 months after its completion only in the presence of premenopausal estradiol levels (and during this period, patients were allowed to receive tamoxifen alone). The survival benefits observed for the primary comparison (tamoxifen plus OFS vtamoxifen alone) were more evident in patients with prior exposure to chemotherapy and in women younger than age 35 years. ${ }^{30}$ In the ASTRRA trial, OFS was administered for 2 years; only patients who received prior chemotherapy were eligible if they remained premenopausal or resumed ovarian function (defined by menstrual history or FSH levels) within 2 years after its completion. ${ }^{33}$

By providing the most profound suppression of hormone serum levels, ${ }^{34,35}$ the combination of OFS plus an Al represents another step in escalating adjuvant endocrine treatment. Three trials assessed this combination (Table 2). 30,36,37 $^{-1}$ With a two-by-two factorial design, the ABCSG-12 study randomly allocated patients to receive OFS plus anastrozole or tamoxifen with or without zoledronic acid. Patients who received OFS plus anastrozole had similar DFS but worse OS compared with those who received OFS plus tamoxifen. ${ }^{36}$ Notably, adjuvant endocrine treatment was administered for 3 years, half of the study population also received adjuvant bisphosphonates, and low-risk patients were included (75\% with primary tumors $\leq 2 \mathrm{~cm}, 66 \%$ with node-negative disease, and only $5 \%$ with prior exposure to neoadjuvant chemotherapy [adjuvant cytotoxic therapy was not allowed]). ${ }^{38}$ TEXT randomly allocated premenopausal patients with hormone receptor-positive tumors to 5 years of OFS plus tamoxifen or exemestane..$^{39} \mathrm{As}$ in SOFT, patients were allowed to receive prior chemotherapy but, when administered $(60 \%$ 


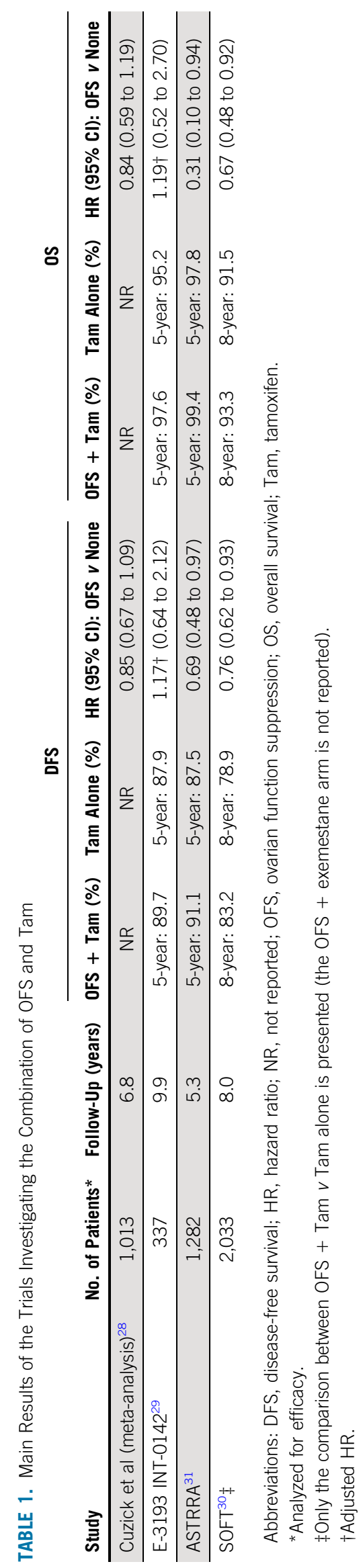




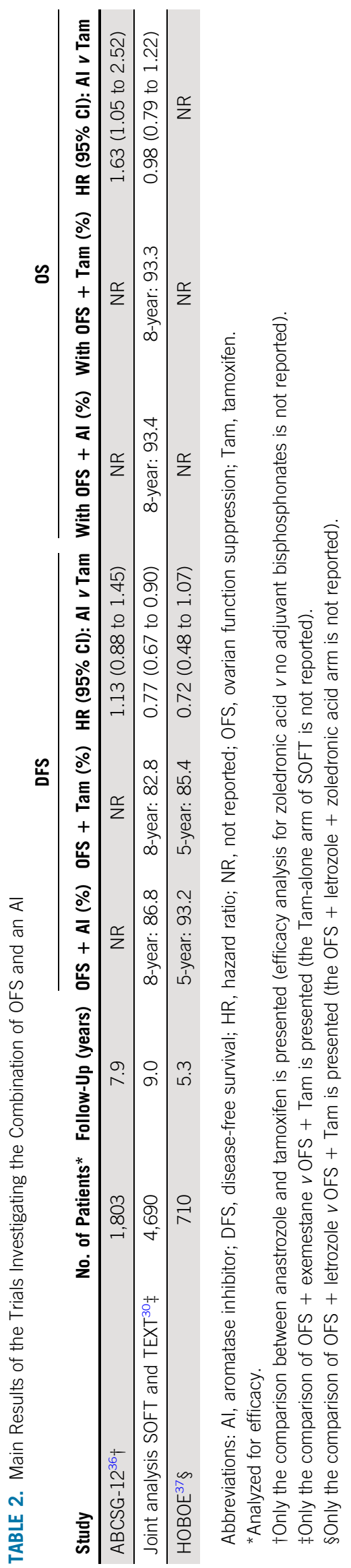


of the study population), OFS had to be started concomitantly with cytotoxic therapy. Patients not receiving chemotherapy started oral endocrine therapy 6-8 weeks after GnRHa initiation. In the joint analysis of TEXT and SOFT, OFS plus exemestane was superior in terms of DFS to OFS plus tamoxifen without difference in OS. ${ }^{30}$ The HOBOE trial was a three-arm study that randomly allocated premenopausal patients with hormone receptor-positive tumors to 5 years of OFS plus tamoxifen or letrozole or letrozole plus zoledronic acid. $^{37}$ The majority of patients $(63 \%)$ received prior chemotherapy. The DFS benefit observed with OFS plus letrozole over tamoxifen was clinically meaningful but reached statistical significance only when combined with adjuvant bisphosphonates. No difference in OS was observed. ${ }^{37}$

On the basis of these data, current guidelines recommend the use of tamoxifen alone for women with low-risk of disease recurrence. For all other patients, particularly those deemed at sufficient risk to justify chemotherapy use, OFS should be considered but without clear preference and guidance on when to combine it with tamoxifen or an Al. ${ }^{4,13,40-42}$

Considering the impact of the toxicity profiles of the different treatments on the patients' quality of life, the available options should be clearly discussed with all women and may influence the final choice (Table 3). ${ }^{29,43,44}$ Notably, up to $20 \%-25 \%$ of patients in the different trials opted for early cessation of endocrine treatment, with higher risk of nonadherence noted in women younger than age 35 years. ${ }^{45}$ Hence, continuous monitoring of adverse events and compliance is crucial; switching to a different option when the ongoing therapy is not tolerated should be preferred over treatment cessation.

When counseling patients on the risk/benefit ratio of the proposed adjuvant treatments, together with the toxicity profile, it is crucial to provide precise information of individual prognosis and expected benefit of the proposed therapies. For premenopausal women with hormone receptor-positive/HER2-negative tumors, refining these estimations would be crucial not only to decide about chemotherapy use but also to distinguish between the different available adjuvant endocrine treatment options.

\section{Refining Prognostication of Patients With Hormone Receptor-Positive/HER2-Negative Breast Cancer}

Indication for adjuvant chemotherapy before endocrine treatment in patients with hormone receptor-positive/ HER2-negative tumors represents one of the most challenging decisions in the breast cancer field. This is even more complex in premenopausal women because of the additional potential age-related negative consequences of chemotherapy.

In premenopausal patients with hormone receptorpositive/HER2-negative breast cancer without nodal involvement, a secondary analysis of TAILORx has recently provided important guidance on estimating chemotherapy benefit using the 21-gene assay. ${ }^{22}$ Although no chemotherapy benefit is expected with an RS of $0-15$, some benefit was observed in women age 50 years or younger with an RS of $16-25 .^{46}$ This benefit was mostly evident in premenopausal women older than age 45 years, suggesting a potential endocrine effect of chemotherapy mediated by the development of premature ovarian insufficiency, an adverse effect happening more frequently among older premenopausal women. ${ }^{22}$ Additional dissection of the TAILORx cohort of women age 50 years and younger with an RS of 16 to 25 according to their clinical risk (defined by tumor size and histologic grade) showed a significant chemotherapy benefit in patients with an RS of 21-25 irrespective of their clinical risk (estimated absolute gain in distant recurrence at 9 years between $6.4 \%$ and $8.7 \%$ ) as well as in those with an RS of 16-20 and high clinical risk (estimated absolute gain in distant recurrence at 9 years of $6.5 \%$ ). Importantly, most of premenopausal patients received tamoxifen alone as adjuvant endocrine treatment; the absolute benefit observed with chemotherapy in TAILORx appears comparable to the one expected with OFS plus Al compared with tamoxifen alone in TEXT and SOFT. In patients with an RS of 16-20 and low clinical risk, no chemotherapy benefit was observed (estimated absolute gain in distant recurrence at 9 years of $-0.2 \%)^{22}$

Despite providing robust prognostic information and prediction of chemotherapy benefit in patients with node-negative

TABLE 3. Main Results of the Patient-Reported Outcomes With the Different Available Adjuvant Endocrine Treatment Options for Premenopausal Patients OFS + Tam ( $v$ Tam alone)

OFS + Al ( $v$ OFS + Tam)

\begin{tabular}{ll}
\hline $\begin{array}{l}\downarrow \text { health-related QoL } \\
\uparrow \text { endocrine symptoms (hot flushes, sweats, sleep disturbance) }\end{array}$ & $\approx$ health-related QoL \\
\hline $\begin{array}{l}\downarrow \text { sexual functioning (vaginal dryness, difficulties in becoming } \\
\text { aroused, decline in sexual interest) }\end{array}$ & $\begin{array}{l}\downarrow \text { hot flushes and sweats } \\
\begin{array}{l}\downarrow \text { bone and joint pain } \\
\downarrow \text { coping effort }\end{array}\end{array}$ \\
\hline
\end{tabular}

$\uparrow$ treatment burden

Abbreviations: Al, aromatase inhibitor; OFS, ovarian function suppression; QoL, quality of life; Tam, tamoxifen. 
hormone receptor-positive/HER2-negative breast cancer, genomic tests have not been developed to select the most appropriate adjuvant endocrine treatment. The study by Pagani et $a l,{ }^{47}$ which accompanies this article, provides important data to guide and improve adjuvant endocrine treatment tailoring in premenopausal women with hormone receptor-positive/HER2-negative tumors. A secondary analysis of SOFT and TEXT was conducted to refine the estimates of the absolute magnitude of endocrine treatment effects in preventing distant recurrences according to patient prognosis. Classic clinicopathologic features (ie, age, tumor size, nodal status, grade, estrogen receptor level, progesterone receptor level, and Ki-67 expression level) were combined into a single continuous value named composite risk (ie, Regan risk score). Using the STEPP methodology, absolute treatment effects were investigated across the continuum of composite risk for 4,891 premenopausal women with hormone receptor-positive/HER2-negative breast cancer. The median follow-up was 9 years in TEXT and was 8 years in SOFT. The main messages from this analysis are the following: (1) in high-risk patients after chemotherapy, the higher the risk of recurrence, the larger the benefit expected with OFS plus exemestane compared with OFS plus tamoxifen or tamoxifen alone (estimated absolute gain in distant recurrence at 8 years up to 15\%); (2) in low-risk patients without prior chemotherapy (overall 8-year freedom from distant recurrence ranging between 97\% and 99\%), there were no clinically relevant differences between the three endocrine treatment options (estimated absolute gain in distant recurrence at 8 years with OFS of approximately 1\%); (3) in intermediate-risk patients, the benefit of OFS plus exemestane compared with OFS plus tamoxifen was more evident in the cohort of patients who did not receive chemotherapy (estimated absolute gain in distant recurrence at 8 years of up to $4 \%$ ). Notably, selection of adjuvant chemotherapy in SOFT and TEXT was based on the same classic clinicopathologic features included in the Regan risk score (at that time, genomic tests were not widely available). On the basis of these data, together with the recent secondary analysis of TAILORX, clinicians now have valid tools to individualize and tailor the counseling of premenopausal women with hormone receptor-positive/ HER2-negative breast cancer on the risk/benefit ratio of the proposed adjuvant treatments (Fig 1). Results of the RxPONDER trial (ClinicalTrials.gov identifier: NCT01272037) investigating the benefit of adding chemotherapy to different endocrine treatments in patients with one to three positive nodes and an RS of 25 or lower are awaited to further refine the counseling of the intermediate-risk population.

\section{Practical Issues and Unanswered Questions}

When the recent evidence on adjuvant endocrine treatment in premenopausal women is translated into clinical care, some practical issues and unanswered questions arise.

In premenopausal women receiving an $\mathrm{Al}$ as oral endocrine therapy, complete OFS must be obtained (although this is not required for tamoxifen). Combining a GnRHa with an $\mathrm{Al}$ is associated with more profound OFS than a GnRHa plus tamoxifen. ${ }^{34,35}$ However, incomplete OFS is expected in approximately $20 \%$ of the patients receiving a $\mathrm{GnRHa}$ plus Al. ${ }^{35}$ No prior exposure to chemotherapy, very young age, and high body mass index are potential risk factors for incomplete OFS to be considered during treatment decision making. ${ }^{35,48}$ For the same reason, a GnRHa plus an $\mathrm{Al}$ is not the preferred choice for patients with compliance issues at risk for nonadherence to monthly injections. Importantly, when a GnRHa plus an Al is chosen, a continuous monitoring of treatment adherence and the possible occurrence of physiologic changes that suggest potential recovery of ovarian function (eg, cyclical fluctuation of climacteric symptoms and menstrual resumption) are crucial. Estradiol and FSH monitoring during treatment can be considered. ${ }^{40,42,49}$ In the case of incomplete OFS during treatment with a GnRHa plus an Al, switching to tamoxifen (or bilateral oophorectomy) should be considered. The use of $\mathrm{GnRH}$ antagonists may partly overcome concerns about inadequate $\mathrm{OFS}^{50}$; future studies are awaited to define the role of $\mathrm{GnRH}$ antagonists in the adjuvant endocrine treatment of premenopausal patients.

The need to obtain complete OFS in patients receiving a GnRHa plus an Al may also impact the timing to start pharmacologic OFS and oral endocrine therapy. When chemotherapy is administered, concurrent administration of a GnRHa is safe ${ }^{51,52}$ and is an effective strategy to reduce the risk of premature ovarian insufficiency. ${ }^{52,53}$ This approach is endorsed by current guidelines. ${ }^{4,40,42,54,55}$ Starting OFS before chemotherapy and continuing the treatment up to 5 years would also facilitate the choice of the oral endocrine therapy partner. If OFS is not given concurrently and chemotherapy-induced amenorrhea occurs, ovarian function should be constantly monitored, because it can resume even beyond 2 years after chemotherapy completion ${ }^{56,57}$; an Al alone in this setting should be avoided because of the significant risk of ovarian function recovery. ${ }^{58}$

Among the potential long-term negative consequences of OFS, especially when given concomitantly with an Al, particular attention should be given to bone loss. By reducing risk of skeletal complications and potentially improving survival outcomes, ${ }^{36-38,59}$ prophylactic use of bisphosphonates can be considered in patients with treatment-related bone loss. ${ }^{42,60,61}$

Because of the significant risk of recurrence beyond 5 years after diagnosis, ${ }^{62}$ extended adjuvant endocrine treatment is the standard of care for many patients with hormone receptor-positive breast cancer. ${ }^{63}$ Premenopausal women have a higher risk of developing late recurrences ${ }^{62}$ and appear to derive the greater benefit from extended adjuvant endocrine treatment. ${ }^{64}$ Tamoxifen alone may be considered in women who remain premenopausal after the first 5 years of therapy, and an $\mathrm{Al}$ alone may be considered in those who become postmenopausal. ${ }^{63}$ However, no 


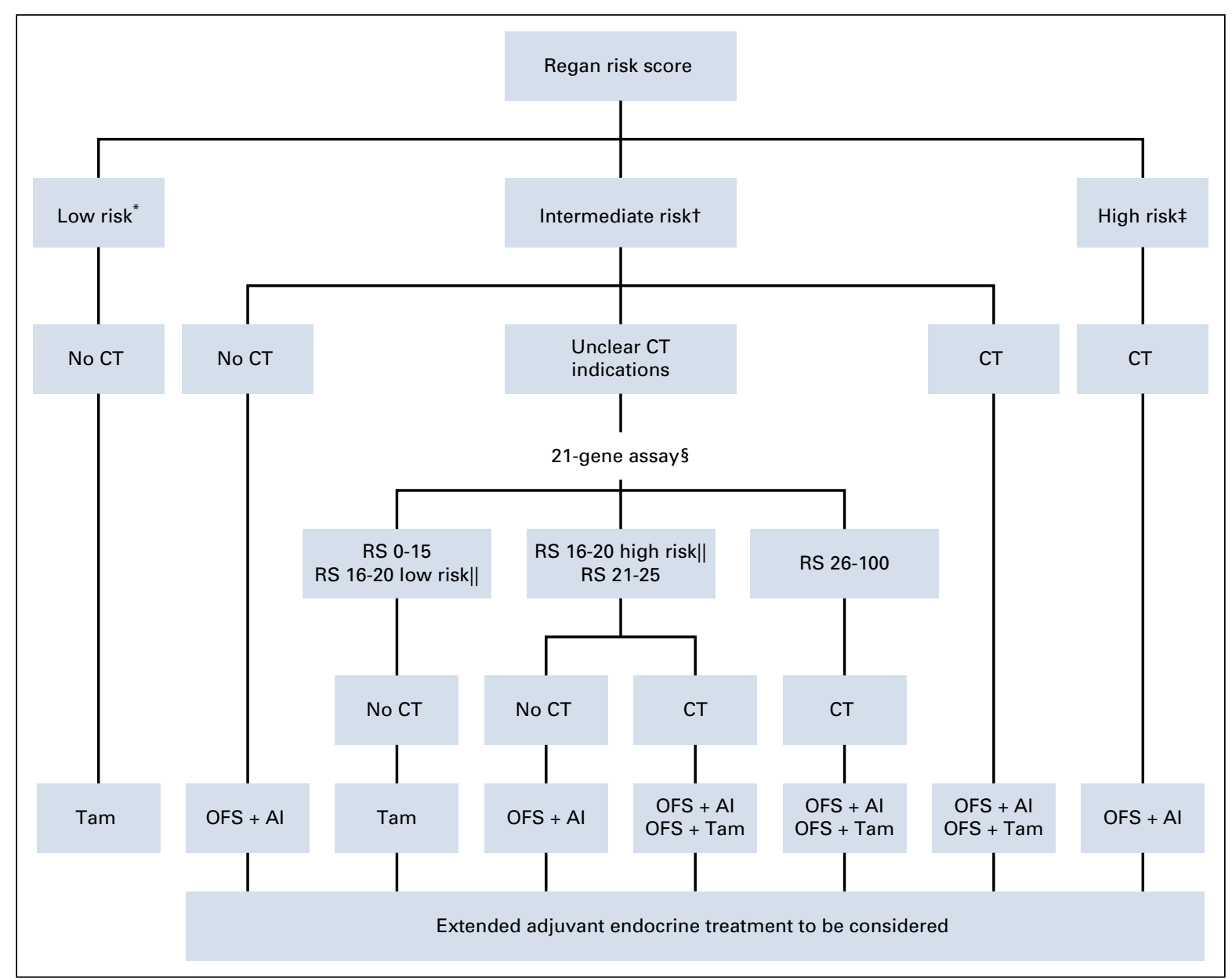

FIG 1. Proposed algorithm for the adjuvant treatment of premenopausal women with hormone receptor-positive/HER2-negative breast cancer, integrating the results obtained with the Regan risk score and the 21-gene assay for the intermediate-risk group. $\left({ }^{*}\right)$ Example of clinical case 1 (age 41 years; grade 1; pT1c pNO; estrogen receptor [ER] $\geq 50 \%$; progesterone receptor [PR] $\geq 50 \%$; Ki-67, 11\%). ( $\dagger$ ) Examples of clinical case 2 (age 39 years; grade 2; pT1c pNO; ER approximately 50\%; PR approximately 50\%; RS of 21) and clinical case 3 (age 40 years; grade 2; pT1c pN1a; ER approximately 50\%; PR approximately 50\%). ( $\ddagger$ ) Example of clinical case 4 (age 39 years; grade 3; pT2 pN1a; ER, 95\%; PR, 40\%). (§) TAILORx provided data for patients with node-negative disease only; results of RxPONDER in patients with one to three positive nodes are awaited. (II) Low-risk was defined as tumor $\leq 3 \mathrm{~cm}$ and grade 1 or tumor $\leq 2 \mathrm{~cm}$ and grade 2 or tumor $\leq 1 \mathrm{~cm}$ and grade 3 (and when the low-risk criteria were not met, the clinical risk was defined as high). Al, aromatase inhibitor; CT, chemotherapy; OFS, ovarian function suppression; RS, recurrence score; Tam, tamoxifen.

evidence exists to decide the optimal approach for extending treatment in patients receiving OFS in the first 5 years. ${ }^{65}$

More evidence is needed to define the optimal adjuvant endocrine treatment of premenopausal (and postmenopausal) patients with hormone receptorpositive/HER2-positive breast cancer. ${ }^{66-68}$ Subgroup analysis of the SOFT, TEXT, and HOBOE trials showed heterogeneity of treatment effect according to HER2 status and noted an apparent greater benefit from the addition of OFS and a lesser efficacy of Als in patients with HER2-positive disease. ${ }^{30,37}$ However, no strong conclusions can be derived because of the limited number of patients included who had HER2-positive disease and the lack of trastuzumab use for many of them. The Regan risk score did not include patients with HER2-positive disease. ${ }^{47}$

\section{SUGGESTED APPROACHES TO MANAGEMENT}

Several risk prediction models based on traditional clinicopathologic features have been developed to refine disease prognostication for patients and aid in treatment decision making. ${ }^{69}$ However, the performance of these models in some patient subgroups, including premenopausal women, has been shown to be suboptimal. ${ }^{69,70}$ In addition, the adjuvant endocrine treatment landscape for premenopausal women has been revolutionized so that reliable predictions about the expected benefit from adjuvant therapies in these patients have become difficult. By improving the stratification of patients' prognosis and the 
quantification of treatment benefit, the Regan risk score (for which a Web application will be available online soon) has a major impact on clinical practice by allowing clearer counseling of the pros and cons of the proposed options; thus, the Regan risk score may be a valid tool to increase women's motivation to follow the prescribed treatment. ${ }^{47}$ Recent data from TAILORx also suggest the possibility to better optimize chemotherapy indications for the cohort of patients with nodenegative disease within the gray zone of the intermediate-risk category by the Regan risk score. ${ }^{22}$ Taken together, these data have allowed another step toward improving adjuvant endocrine treatment tailoring for premenopausal patients (Fig 1).

Clinical case 1 reports about a patient who, after breastconserving surgery, was diagnosed with a disease that can be defined as low risk (stage I, lymph node negative, grade 1 , estrogen receptor/progesterone receptor strongly positive, Ki67 low) according to the Regan risk score without the need for genomic tests to make adjuvant treatment decisions. After multidisciplinary discussion, adjuvant radiotherapy and endocrine therapy with tamoxifen alone for 5 years was recommended.

Clinical case 2 is in the gray zone of the intermediate-risk category (stage I, lymph node negative, grade 2 , estrogen receptor/progesterone receptor intermediate expression) by the Regan risk score, for which the 21-gene assay results is of added value to optimize disease management; with a RS of 21, we opted for anthracycline-free chemotherapy followed by OFS plus tamoxifen after discussion of the

\section{AFFILIATIONS}

${ }^{1}$ IRCCS Ospedale Policlinico San Martino, Genoa, Italy

${ }^{2}$ University of Genova, Genoa, Italy

${ }^{3}$ Istituto Nazionale Tumori, IRCCS Fondazione G. Pascale, Naples, Italy

\section{CORRESPONDING AUTHOR}

Matteo Lambertini, MD, PhD, IRCCS Ospedale Policlinico San Martino, University of Genova, Largo Rosanna Benzi 10, 16132 Genoa, Italy; Twitter: @matteolambe, @SanMartino_Ge, and @UniGenova; e-mail: matteo.lambertini@unige.it. different toxicity profile of the two oral endocrine agents. However, during patient counseling, the potential overtreatment with chemotherapy administration and the possibility that the same benefit expected with adjuvant treatments could have been obtained by OFS plus an Al without prior chemotherapy was discussed.

Clinical case 3 falls also within the intermediate-risk category by the Regan risk score (stage II, one of three sentinel lymph nodes positive, grade 2, and estrogen receptor/progesterone receptor intermediate expression); the patient came to our center after receiving anthracycline-free chemotherapy. She resumed ovarian function after chemotherapy. We suggested the use of OFS with tamoxifen or an Al; after discussing the different toxicity profile of the two oral endocrine agents, the Al was preferred and started after the second administration of a GnRHa with estradiol and FSH monitoring every 3-6 months at least during the first year of treatment.

Clinical case 4 describes a high-risk patient according to the Regan risk score (stage II, three positive lymph nodes, grade 3, estrogen receptor strongly positive, progesterone receptor intermediate expression); without the need for genomic tests to make adjuvant treatment decisions, anthracycline- and taxane-based chemotherapy was recommended. The patient has three children but was concerned about risk of chemotherapy-induced premature ovarian insufficiency. A GnRHa was started 1 week before chemotherapy; an Al was added after the end of cytotoxic therapy with estradiol and FSH monitoring.

AUTHORS' DISCLOSURES OF POTENTIAL CONFLICTS OF INTEREST AND DATA AVAILABILITY STATEMENT

Disclosures provided by the authors and data availability statement (if applicable) are available with this article at DOI https://doi.org/10.1200/ JCO.19.02242

\section{AUTHOR CONTRIBUTIONS}

Conception and design: All authors

Data analysis and interpretation: All authors

Collection and assembly of data: All authors

Manuscript writing: All authors

Final approval of manuscript: All authors

Accountable for all aspects of the work: All authors

\section{REFERENCES}

1. Siegel RL, Miller KD, Jemal A: Cancer statistics, 2019. CA Cancer J Clin 69:7-34, 2019

2. Ghiasvand R, Adami H-O, Harirchi I, et al: Higher incidence of premenopausal breast cancer in less developed countries: Myth or truth? BMC Cancer 14:343, 2014

3. Azim HA, Jr., Partridge AH: Biology of breast cancer in young women. Breast Cancer Res 16:427, 2014

4. Burstein HJ, Curigliano G, Loibl S, et al: Estimating the benefits of therapy for early stage breast cancer: The St Gallen international consensus guidelines for the primary therapy of early breast cancer 2019. Ann Oncol [epub ahead of print on August 2, 2019] https://doi.org/10.1093/annonc/mdz235

5. Howard-Anderson J, Ganz PA, Bower JE, et al: Quality of life, fertility concerns, and behavioral health outcomes in younger breast cancer survivors: A systematic review. J Natl Cancer Inst 104:386-405, 2012

6. Ruddy KJ, Gelber SI, Tamimi RM, et al: Prospective study of fertility concerns and preservation strategies in young women with breast cancer. J Clin Oncol 32: 1151-1156, 2014

7. Lambertini M, Fontana V, Massarotti C, et al: Prospective study to optimize care and improve knowledge on ovarian function and/or fertility preservation in young breast cancer patients: Results of the pilot phase of the pregnancy and fertility (PREFER) study. Breast 41:51-56, 2018 
8. Ruggeri M, Pagan E, Bagnardi V, et al: Fertility concerns, preservation strategies and quality of life in young women with breast cancer: Baseline results from an ongoing prospective cohort study in selected European Centers. Breast 47:85-92, 2019

9. Partridge AH, Hughes ME, Warner ET, et al: Subtype-dependent relationship between young age at diagnosis and breast cancer survival. J Clin Oncol 34: 3308-3314, 2016

10. Sopik V, Sun P, Narod SA: The prognostic effect of estrogen receptor status differs for younger versus older breast cancer patients. Breast Cancer Res Treat 165 : 391-402, 2017

11. Yoon TI, Hwang U-K, Kim ET, et al: Survival improvement in hormone-responsive young breast cancer patients with endocrine therapy. Breast Cancer Res Treat 165:311-320, 2017

12. Burstein $\mathrm{HJ}$, Temin S, Anderson $\mathrm{H}$, et al: Adjuvant endocrine therapy for women with hormone receptor-positive breast cancer: American Society of Clinical Oncology clinical practice guideline focused update. J Clin Oncol 32:2255-2269, 2014

13. Burstein $\mathrm{HJ}$, Lacchetti C, Anderson $\mathrm{H}$, et al: Adjuvant endocrine therapy for women with hormone receptor-positive breast cancer: American Society of Clinical Oncology clinical practice guideline update on ovarian suppression. J Clin Oncol 34:1689-1701, 2016

14. Huiart L, Bouhnik A-D, Rey D, et al: Early discontinuation of tamoxifen intake in younger women with breast cancer: Is it time to rethink the way it is prescribed? Eur J Cancer 48:1939-1946, 2012

15. Wassermann J, Gelber SI, Rosenberg SM, et al: Nonadherent behaviors among young women on adjuvant endocrine therapy for breast cancer. Cancer 125 : 3266-3274, 2019

16. Llarena NC, Estevez SL, Tucker SL, et al: Impact of fertility concerns on tamoxifen initiation and persistence. J Natl Cancer Inst 107:djv202, 2015

17. Pagani O, Ruggeri M, Manunta S, et al: Pregnancy after breast cancer: Are young patients willing to participate in clinical studies? Breast 24:201-207, 2015

18. Azim HA, Jr, Nguyen B, Brohée S, et al: Genomic aberrations in young and elderly breast cancer patients. BMC Med 13:266, 2015

19. Kan Z, Ding Y, Kim J, et al: Multi-omics profiling of younger Asian breast cancers reveals distinctive molecular signatures. Nat Commun 9:1725, 2018

20. Liao S, Hartmaier RJ, McGuire KP, et al: The molecular landscape of premenopausal breast cancer. Breast Cancer Res 17:104, 2015

21. Cardoso F, van't Veer LJ, Bogaerts J, et al: 70-gene signature as an aid to treatment decisions in early-stage breast cancer. N Engl J Med 375:717-729, 2016

22. Sparano JA, Gray RJ, Ravdin PM, et al: Clinical and genomic risk to guide the use of adjuvant therapy for breast cancer. N Engl J Med 380:2395-2405, 2019

23. Lambertini M, Viglietti G, de Azambuja E: Controversies in oncology: Which adjuvant endocrine therapy is to be given to premenopausal patients with hormone receptor-positive breast cancer? ESMO Open 3:e000350, 2018

24. Regan MM, Fleming GF, Walley B, et al: Adjuvant systemic treatment of premenopausal women with hormone receptor-positive early breast cancer: Lights and shadows. J Clin Oncol 37:862-866, 2019

25. Davies C, Godwin J, Gray R, et al: Relevance of breast cancer hormone receptors and other factors to the efficacy of adjuvant tamoxifen: Patient-level metaanalysis of randomised trials. Lancet 378:771-784, 2011

26. Zhao J, Liu J, Chen $\mathrm{K}$, et al: What lies behind chemotherapy-induced amenorrhea for breast cancer patients: A meta-analysis. Breast Cancer Res Treat 145 : 113-128, 2014

27. Lambertini M, Campbell C, Bines J, et al: Adjuvant anti-HER2 therapy, treatment-related amenorrhea, and survival in premenopausal HER2-positive early breast cancer patients. J Natl Cancer Inst 111:86-94, 2019

28. Cuzick J, Ambroisine L, Davidson N, et al: Use of luteinising-hormone-releasing hormone agonists as adjuvant treatment in premenopausal patients with hormone-receptor-positive breast cancer: A meta-analysis of individual patient data from randomised adjuvant trials. Lancet 369:1711-1723, 2007

29. Tevaarwerk AJ, Wang M, Zhao F, et al: Phase III comparison of tamoxifen versus tamoxifen plus ovarian function suppression in premenopausal women with node-negative, hormone receptor-positive breast cancer (E-3193, INT-0142): A trial of the Eastern Cooperative Oncology Group. J Clin Oncol 32:3948-3958, 2014

30. Francis PA, Pagani O, Fleming GF, et al: Tailoring adjuvant endocrine therapy for premenopausal breast cancer. N Engl J Med 379:122-137, 2018

31. Kim H-A, Lee JW, Nam SJ, et al: Adding ovarian suppression to tamoxifen for premenopausal breast cancer: A randomized phase III trial. J Clin Oncol 10.1200/ JCO.19.00126 [epub ahead of print on September 16, 2019]

32. Francis PA, Regan MM, Fleming GF, et al: Adjuvant ovarian suppression in premenopausal breast cancer. N Engl J Med 372:436-446, 2015

33. Kim H-A, Ahn SH, Nam SJ, et al: The role of the addition of ovarian suppression to tamoxifen in young women with hormone-sensitive breast cancer who remain premenopausal or regain menstruation after chemotherapy (ASTRRA): Study protocol for a randomized controlled trial and progress. BMC Cancer 16:319, 2016

34. Rossi E, Morabito A, De Maio E, et al: Endocrine effects of adjuvant letrozole + triptorelin compared with tamoxifen + triptorelin in premenopausal patients with early breast cancer. J Clin Oncol 26:264-270, 2008

35. Bellet M, Gray KP, Francis PA, et al: Twelve-month estrogen levels in premenopausal women with hormone receptor-positive breast cancer receiving adjuvant triptorelin plus exemestane or tamoxifen in the suppression of ovarian function trial (SOFT): The SOFT-EST substudy. J Clin Oncol 34:1584-1593, 2016

36. Gnant $\mathrm{M}$, Mlineritsch B, Stoeger $\mathrm{H}$, et al: Zoledronic acid combined with adjuvant endocrine therapy of tamoxifen versus anastrozole plus ovarian function suppression in premenopausal early breast cancer: Final analysis of the Austrian Breast and Colorectal Cancer Study Group Trial 12. Ann Oncol 26:313-320, 2015

37. Perrone F, De Laurentiis M, De Placido S, et al: Adjuvant zoledronic acid and letrozole plus ovarian function suppression in premenopausal breast cancer: HOBOE phase 3 randomised trial. Eur J Cancer 118:178-186, 2019

38. Gnant M, Mlineritsch B, Schippinger W, et al: Endocrine therapy plus zoledronic acid in premenopausal breast cancer. N Engl J Med 360:679-691, 2009

39. Pagani O, Regan MM, Walley BA, et al: Adjuvant exemestane with ovarian suppression in premenopausal breast cancer. N Engl J Med 371:107-118, 2014

40. Paluch-Shimon S, Pagani O, Partridge AH, et al: ESO-ESMO 3rd international consensus guidelines for breast cancer in young women (BCY3). Breast 35: 203-217, 2017

41. Gori S, Puglisi F, Cinquini M, et al: Adjuvant endocrine therapy in premenopausal patients with hormone receptor-positive early breast cancer: Evidence evaluation and GRADE recommendations by the Italian Association of Medical Oncology (AIOM). Eur J Cancer 99:9-19, 2018

42. Cardoso F, Kyriakides S, Ohno S, et al: Early breast cancer: ESMO clinical practice guidelines for diagnosis, treatment and follow-up. Ann Oncol 30:1194-1220, 2019

43. Ribi K, Luo W, Bernhard J, et al: Adjuvant tamoxifen plus ovarian function suppression versus tamoxifen alone in premenopausal women with early breast cancer: Patient-reported outcomes in the suppression of ovarian function trial. J Clin Oncol 34:1601-1610, 2016

44. Bernhard J, Luo W, Ribi K, et al: Patient-reported outcomes with adjuvant exemestane versus tamoxifen in premenopausal women with early breast cancer undergoing ovarian suppression (TEXT and SOFT): A combined analysis of two phase 3 randomised trials. Lancet Oncol 16:848-858, 2015 
45. Saha P, Regan MM, Pagani O, et al: Treatment efficacy, adherence, and quality of life among women younger than 35 years in the International Breast Cancer Study Group TEXT and SOFT adjuvant endocrine therapy trials. J Clin Oncol 35:3113-3122, 2017

46. Sparano JA, Gray RJ, Makower DF, et al: Adjuvant chemotherapy guided by a 21-gene expression assay in breast cancer. N Engl J Med 379:111-121, 2018

47. Pagani $\mathrm{O}$, Francis PA, Fleming GF, et al: Absolute improvements in freedom from distant recurrence to tailor adjuvant endocrine therapies for premenopausal women with breast cancer: Results from TEXT and SOFT. J Clin Oncol doi:10.1200/JC0.2019.18.01967)

48. Pfeiler G, Königsberg R, FesI C, et al: Impact of body mass index on the efficacy of endocrine therapy in premenopausal patients with breast cancer: An analysis of the prospective ABCSG-12 trial. J Clin Oncol 29:2653-2659, 2011

49. Papakonstantinou A, Foukakis T, Rodriguez-Wallberg KA, et al: Is estradiol monitoring necessary in women receiving ovarian suppression for breast cancer? J Clin Oncol 34:1573-1579, 2016

50. Dellapasqua S, Gray KP, Munzone E, et al: Neoadjuvant degarelix versus triptorelin in premenopausal patients who receive letrozole for locally advanced endocrine-responsive breast cancer: A randomized phase II trial. J Clin Oncol 37:386-395, 2019

51. Regan MM, Walley BA, Francis PA, et al: Concurrent and sequential initiation of ovarian function suppression with chemotherapy in premenopausal women with endocrine-responsive early breast cancer: An exploratory analysis of TEXT and SOFT. Ann Oncol 28:2225-2232, 2017

52. Lambertini M, Moore HCF, Leonard RCF, et al: Gonadotropin-releasing hormone agonists during chemotherapy for preservation of ovarian function and fertility in premenopausal patients with early breast cancer: A systematic review and meta-analysis of individual patient-level data. J Clin Oncol 36:1981-1990, 2018

53. Lambertini M, Ceppi M, Poggio F, et al: Ovarian suppression using luteinizing hormone-releasing hormone agonists during chemotherapy to preserve ovarian function and fertility of breast cancer patients: A meta-analysis of randomized studies. Ann Oncol 26:2408-2419, 2015

54. Lambertini M, Cinquini M, Moschetti I, et al: Temporary ovarian suppression during chemotherapy to preserve ovarian function and fertility in breast cancer patients: A GRADE approach for evidence evaluation and recommendations by the Italian Association of Medical Oncology. Eur J Cancer 71:25-33, 2017

55. Oktay K, Harvey BE, Partridge AH, et al: Fertility preservation in patients with cancer: ASCO clinical practice guideline update. J Clin Oncol 36:1994-2001, 2018

56. Lambertini M, Boni L, Michelotti A, et al: Ovarian suppression with triptorelin during adjuvant breast cancer chemotherapy and long-term ovarian function, pregnancies, and disease-free survival: A randomized clinical trial. JAMA 314:2632-2640, 2015

57. Moore HCF, Unger JM, Phillips K-A, et al: Final analysis of the prevention of early menopause study (POEMS)/SWOG Intergroup S0230. J Natl Cancer Inst 111: 210-213, 2019

58. van Hellemond IEG, Vriens IJH, Peer PGM, et al: Ovarian function recovery during anastrozole in breast cancer patients with chemotherapy-induced ovarian function failure. J Natl Cancer Inst 109:djx074, 2017

59. Nuzzo F, Gallo C, Lastoria S, et al: Bone effect of adjuvant tamoxifen, letrozole or letrozole plus zoledronic acid in early-stage breast cancer: The randomized phase 3 HOBOE study. Ann Oncol 23:2027-2033, 2012

60. Hadji P, Coleman RE, Wilson C, et al: Adjuvant bisphosphonates in early breast cancer: Consensus guidance for clinical practice from a European panel. Ann Oncol 27:379-390, 2016

61. Dhesy-Thind S, Fletcher GG, Blanchette PS, et al: Use of adjuvant bisphosphonates and other bone-modifying agents in breast cancer: A Cancer Care Ontario and American Society of Clinical Oncology clinical practice guideline. J Clin Oncol 35:2062-2081, 2017

62. Pan H, Gray R, Braybrooke J, et al: 20-year risks of breast-cancer recurrence after stopping endocrine therapy at 5 years. N Engl J Med 377:1836-1846, 2017

63. Burstein $\mathrm{HJ}$, Lacchetti $\mathrm{C}$, Anderson $\mathrm{H}$, et al: Adjuvant endocrine therapy for women with hormone receptor-positive breast cancer: ASCO clinical practice guideline focused update. J Clin Oncol 37:423-438, 2019

64. Goss PE, Ingle JN, Martino S, et al: Impact of premenopausal status at breast cancer diagnosis in women entered on the placebo-controlled NCIC CTG MA17 trial of extended adjuvant letrozole. Ann Oncol 24:355-361, 2013

65. Ruddy KJ, DeSantis SD, Barry W, et al: Extended therapy with letrozole and ovarian suppression in premenopausal patients with breast cancer after tamoxifen. Clin Breast Cancer 14:413-416, 2014

66. Bartlett JMS, Ahmed I, Regan MM, et al: HER2 status predicts for upfront Al benefit: A TRANS-AIOG meta-analysis of 12,129 patients from ATAC, BIG 1-98 and TEAM with centrally determined HER2. Eur J Cancer 79:129-138, 2017

67. Dackus GMHE, Jóźwiak K, Sonke GS, et al: Optimal adjuvant endocrine treatment of ER+/HER2+ breast cancer patients by age at diagnosis: A populationbased cohort study. Eur J Cancer 90:92-101, 2018

68. Lambertini M, Campbell C, Gelber RD, et al: Dissecting the effect of hormone receptor status in patients with HER2-positive early breast cancer: Exploratory analysis from the ALTTO (BIG 2-06) randomized clinical trial. Breast Cancer Res Treat 177:103-114, 2019

69. Engelhardt EG, Garvelink MM, de Haes JHCJM, et al: Predicting and communicating the risk of recurrence and death in women with early-stage breast cancer: A systematic review of risk prediction models. J Clin Oncol 32:238-250, 2014

70. Lambertini M, Pinto AC, Ameye L, et al: The prognostic performance of Adjuvant! Online and Nottingham Prognostic Index in young breast cancer patients. Br J Cancer 115:1471-1478, 2016 


\section{Improving Adjuvant Endocrine Treatment Tailoring in Premenopausal Women With Hormone Receptor-Positive Breast Cancer}

The following represents disclosure information provided by authors of this manuscript. All relationships are considered compensated. Relationships are self-held unless noted. I = Immediate Family Member, Inst = My Institution. Relationships may not relate to the subject matter of this manuscript. For more information about ASCO's conflict of interest policy, please refer to www.asco.org/rwc or ascopubs.org/jco/site/ifc.

Matteo Lambertini

Speakers' Bureau: Theramex, Takeda

Francesco Perrone

Honoraria: Bayer, Sandoz, Incyte, Celgene, Bayer, AstraZeneca, Pierre Fabre, Janssen-Cilag

Research Funding: Roche (Inst), Bayer (Inst), AstraZeneca (Inst), Pfizer (Inst), Incyte (Inst)

Travel, Accommodations, Expenses: Bayer

\section{Lucia Del Mastro}

Honoraria: Roche, Pfizer, Ipsen, Lilly, Eisai, Novartis, Takeda

Consulting or Advisory Role: Lilly, Roche, MSD, Novartis, Genomic Health

Travel, Accommodations, Expenses: Roche, Pfizer, Celgene

No other potential conflicts of interest were reported. 